Somnologie 2011 $\cdot 15: 197-198$

DOI 10.1007/s11818-011-0549-2

Online publiziert: 4. Dezember 2011

(c) Springer-Verlag 2011

\author{
J. Fischer \\ früher: Klinik Norderney der DRV-Westfalen, Norderney
}

\title{
Leistungsfähigkeit und Schlaf
}

Eine eingeschränkte Leistungsfähigkeit bei einem Kutscher, die sich in einer ausgeprägten Tagesschläfrigkeit bei massivem Übergewicht äußerte und eindrucksvoll von Charles Dickens in seinen zwischen 1836 und 1837 publizierten Pickwick Papers [1] beschrieben wurde, wurde kennzeichnend für ein schwerwiegendes Krankheitsbild. Übernommen wurde der Name bei der Erstbeschreibung eines Krankheitsbildes, welches mit schwerer Übergewichtigkeit, Herzinsuffizienz, respiratorischer Insuffizienz und ausgeprägter Tagesschläfrigkeit einherging. Ein Patient, der diese Krankheitserscheinungen aufwies, suchte deshalb den amerikanischen Arzt Burwell und seine Kollegen 1956 [2] auf, weil er beim Pokerspiel mit einem „Full House“ in der Hand eingeschlafen war, ohne den Triumph dieses exzellenten Pokerblatts auskosten zu können. Die eingeschränkte Leistungsfähigkeit hat hier nicht nur zu einer Behinderung der beruflichen, sondern auch der sozialen Partizipation geführt. Die pathophysiologischen Zusammenhänge und die Ursache dieses Kranheitsbildes, des sog. Pickwick-Syndroms, blieben noch lange unbekannt.

Erst 1965 konnten Jung u. Kuhlo [3] in polysomnographischen Untersuchungen aufzeigen, dass es während des Schlafs bei diesen Patienten zu Apnoen, bedingt durch einen Verschluss der oberen extrathorakalen Atemwege kommt und die Terminierung dieser Apnoen durch eine Arousalreaktion hervorgerufen wird. Die pathophysiologischen Zusammenhänge bestehen in ausgeprägter Adipositas, hierdurch bedingter alveolärer Hypoven- tilation mit Hyperkapnie und Hypoxämie, schlafbezogenen Atmungsstörungen mit Apnoen und Hypopnoen und die Apnoen terminierenden Arousalreaktionen mit daraus folgender Einschränkung der Erholungsfunktion des Schlafs. Die mit diesem Syndrom einhergehende Einschränkung der mentalen und körperlichen Leistungsfähigkeit konnte erst durch den Einsatz einer drastischen, aber sehr wirksamen Therapie, nämlich das Anlegen eines Tracheostomas 1969 durch Kuhlo et al. [4] bei den betroffenen Patienten verbessert werden. Die Akzeptanz dieser invasiven Therapie durch die Patienten war allerdings eher gering.

\section{( D Die Einschränkung der Leistungsfähigkeit infolge Tagesmüdigkeit stellten wir in vielen Berufen fest}

Durch die geniale Idee von Sullivan et al. [5], die obstruktiven Apnoen und Hypopnoen mit einer nasalen Überdruckbeatmung (n-CPAP) während des Schlafs zu behandeln, gelang es, die therapeutische Situation für die Patienten deutlich zu verbessern. Mittlerweile hatte man festgestellt, dass auch nicht extrem übergewichtige Patienten schlafbezogene Atmungsstörungen mit ausgeprägter Tagesmüdigkeit und Tagesschläfrigkeit aufweisen können und unter Einschränkung der Leistungsfähigkeit leiden. Es ist aufgrund der Untersuchungen der „Sleep Heart Health Study “ [6] bekannt, dass es sich bei dem obstruktiven Schlafapnoe-Syndrom und seinen Folge- und Begleiterkrankungen wie z. B. arterielle Hypertonie, koro- nare Herzkrankheit, Schlaganfall, Diabetes mellitus und chronische obstruktive Lungenerkrankung (COPD) um eine Volkskrankheit handelt.

Die Einschränkung der mentalen und physischen Leistungsfähigkeit infolge der mehr oder weniger stark ausgeprägten Tagesmüdigkeit und Tagesschläfrigkeit konnten wir in den 25 Jahren der Existenz des Schlaflabors und des 50-jährigen Bestehens der Klinik Norderney der Deutschen Rentenversicherung Westfalen auf Norderney bei vielen Patienten mit unterschiedlichsten Berufen feststellen. Ob es ein Richter war, der bei den Verhandlungen einschlief, ein Tischler an der Kreissäge, ein Pastor auf der Kanzel, ein Landwirt, der im Kreis pflügt, ein Chirurg, der während der Operationen einschläft, der Lokomotivführer, Pilot oder Schiffskapitän, Lastwagenfahrer, Bankdirektor, Hausfrau oder Kinder in der Schule, ihnen allen können wir mit einer differenzierten Diagnostik des Schlafs und der Atmung am Tag und in der Nacht und Einleitung einer suffizienten, akzeptablen Therapie wieder zu mehr Leistungsfähigkeit verhelfen und damit ihre Möglichkeiten zur Teilhabe am beruflichen und sozialen Leben erheblich verbessern.

In diesem Heft der „Somnologie“ werden Artikel zur Diagnostik und Therapie von Schlaf und Atmung unter dem Aspekt

Der Autor war Ärztlicher Direktor der Klinik Norderney der DRV-Westfalen, Inhaber des Lehrstuhls für Rehabilitationswissenschaften der Universität Witten/Herdecke und Direktor des Instituts für Rehabilitationsforschung Norderney. 
der Leistungsfähigkeit vom Schulkind bis zum Palliativpatienten dargestellt. Die Basis dafür war ein internationale Symposium zum Thema „Leistungsfähigkeit atmen, arbeiten, schlafen", das am 30. und 31. Mai 2011 auf Norderney stattfand.

Prof. Dr. Jürgen Fischer

\section{Korrespondenzadresse}

Prof. Dr. J. Fischer

früher: Klinik Norderney der DRV-Westfalen 26548 Norderney

jfischerp@aol.com

\section{Literatur}

1. Dickens C (1837) The posthumous papers of the Pickwick Club, containing a faithful record of the perambulations, perils, travels, adventures and sporting transactions of the corresponding members. Chapman \& Hall, London

2. Burwell CS, Robin ED, Whaley RD, Bickelmann AG (1965) Extreme obesity associated with alveolar hypoventilation - A Pickwickian syndrome. Am J Med 21:811-818

3. Jung R, Kuhlo W (1965) Neurophysiological studies of abnormal night sleep and the Pickwickian syndrome. Prog Brain Res 18:140-159

4. Kuhlo W, Doll E, Franck MC (1969) Successful management of Pickwickian syndrome using longterm tracheostomy. Dtsch Med Wochenschr 94:1286-1290

5. Sullivan CE, Berthon-Jones M, Issa FG, Eves L (1981) Reversal of obstructive sleep apnoea by continuous positive airway pressure applied through the nares. Lancet 317:862-865

6. Quan SF, Howard BV, Iber C et al. (1997) The Sleep Heart Health Study: Design rationale and methods. Sleep 20:1077-1085
The editors of Somnologie / Somnology would like to thank all section editors and all members of the editorial advisory board for their continuous support of our journal. Special thanks go to all reviewers of the years 2010 and 2011 for their time and extensive help to improve the quality of submissions:

\section{0}

Arber S.

Brinkmann P.J.

Cassel W.

Clarenbach P.

Danker-Hopfe H.

Eiselt M.

Erler Th.

Fietze I.

Golz M.

Griefahn B.

Groneberg D.

Hein $\mathrm{H}$.

Heitmann J.

Herold J.

Huetten $M$.

Jones R.

Johns M.

Klementz K.

Klösch G.

Kotterba S.

Maurer J.T.

Mittendorf $\mathrm{H}$.

Nissen C.

Orth M.

Penzel T.

Podszus T.

Poli F.

Pollmächer T.

Randerath W. J.

Rasche K.

Raschke F.

Riedl M.

Riemann D.

Rodenbeck A.

Rödel $\mathrm{H}$.

Rühle K.-H.

Saletu B.

Sanner B.

Schädlich S..

Schäfer T.

Schlüter B.

Schulz H.

Steinberg $R$.

Urschitz M.
Van Mark A.

Vogtmann T.

Weeß H.-G.

Weinreich $\mathrm{G}$.

Wiegand G.

Young $P$.

Young $R$.

Zeitlhofer J.

Zulley J.

\section{1}

Becker H.F.

Blau A.

Bosse-Henck A.

Canisius S.

Chouvarda I.

Crönlein T.

Dorow P.

Eiselt $M$.

Fietze I.

Frohnhofen $\mathrm{H}$.

Galetke W.

Geisler P.

Glos M.

Happe S.

Hein $\mathrm{H}$.

Kelmanson

Klösch G.

Köhler D.

Kotterba S.

Mayer G.

Meisel

Müller A.

Oertel W.

Paiva T.

Parrino L.

Randerath W.J.

Rasche K.

Raschke F.

Salih F.

Sauter C.

Sanner B.

Schäfer D.

Scholle AS.

Schulz H.

Schulz R.

Sebert M.

Steiger A.

Tobler I.

Wetter T.

Wessel N.

Young $\mathrm{P}$. 